Technical note

\title{
Characterization of MOSkin detector for in vivo dose verification during Cobalt-60 high dose-rate intracavitary brachytherapy
}

\author{
Zulaikha Jamalludin ${ }^{\mathrm{a}, \mathrm{b}}$, Wei Loong Jong ${ }^{\mathrm{c}}$, Rozita Abdul Malik ${ }^{\mathrm{c}}$, Anatoly Rosenfeld ${ }^{\mathrm{d}}$, \\ Ngie Min Ung ${ }^{\text {, } *}$ \\ ${ }^{a}$ Department of Clinical Oncology, University of Malaya Medical Centre, Kuala Lumpur, Malaysia \\ ${ }^{\mathrm{b}}$ Medical Physics Unit, University of Malaya Medical Centre, Kuala Lumpur, Malaysia

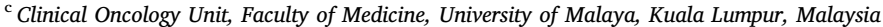 \\ ${ }^{\mathrm{d}}$ Centre for Medical Radiation Physics, University of Wollongong, Wollongong, Australia
}

\begin{abstract}
A B S T R A C T

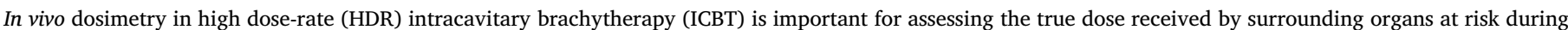

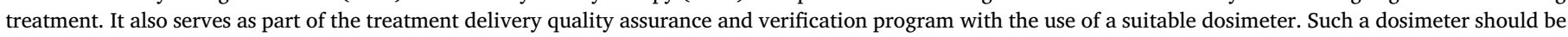

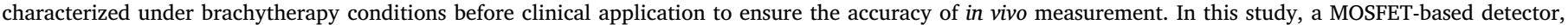

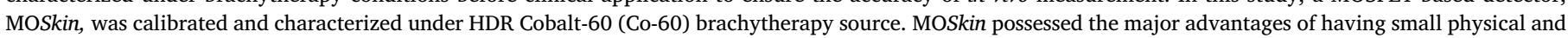

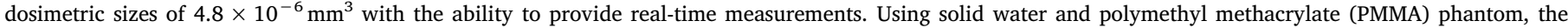

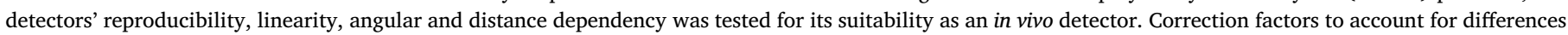

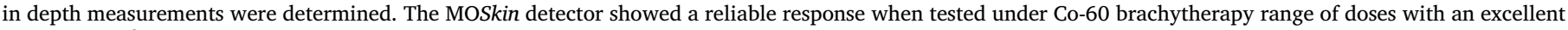

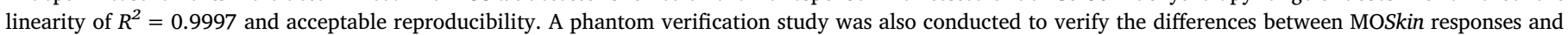

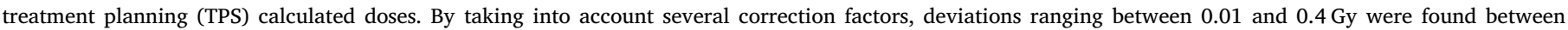

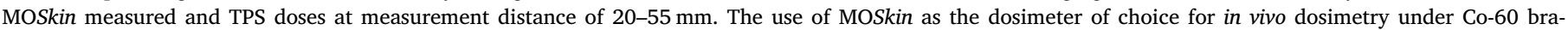
chytherapy condition is feasible.
\end{abstract}

\section{Introduction}

High dose-rate (HDR) intracavitary brachytherapy (ICBT) is part of cervical cancer definitive treatments. The main advantage of ICBT is the delivery of good dose conformality to the tumor while sparing the dose to the surrounding tissues. As the cervix is in close proximity to the rectum and bladder, these organs will indirectly receive part of the dose spillage from the treatment. Clinical correlation between doses in rectal volume with toxicity have been demonstrated in earlier investigations $[1,2]$. Hence it is crucial to monitor the dose received by these organs during treatments. To accomplish this, in vivo dosimetry (IVD) is recommended to be performed during each fraction of ICBT. Any deviations in the dose measured during ICBT allow for modification to the prescribed dose in the subsequent treatment fraction. It also acts as part of, and supplement to, the quality assurance program by providing an independent verification of treatment delivery while having a permanent record of actual organ doses received during treatment.

Development and implementation of IVD in brachytherapy has been conducted using either commercially available or institutional self-developed detectors such as thermoluminescent dosimeters (TLD), diodes, metal-oxide semiconductor field effect transistors (MOSFET), plastic scintillation (PSD), alanine, fiber optic and optically-stimulated luminescence dosimeters (OSLD) [3-10]. Tanderup et al. rated and summarized the advantages and convenience of the abovementioned detectors according to the intrinsic characteristics, ease of handling and availability of the detectors. Among them, MOSFET, OSLD and PSD were categorized as having relatively small volumes that are essential for the high spatial resolution required to provide accurate dose measurements in high dose gradient areas.

MOSFET has the advantage of having features such as dose measurement integration as well as allowing immediate dose readout (realtime measurement) besides having a small volume. Although a commercial MOSFET's size is relatively small compared to other previously mentioned detectors, the volume is still considered to be large for IVD in brachytherapy. The production of micro-MOSFET detectors with a smaller active volume of $0.2 \times 0.2 \times 5 \times 10^{-4} \mathrm{~mm}^{3}$ is expected to overcome the volume averaging problem in brachytherapy dosimetry. Owing to small sizes, micro-MOSFET has been clinically used by inserting the detectors into the brachytherapy catheter to detect dose differences during interstitial brachytherapy of head and neck, breast,

\footnotetext{
* Corresponding author at: Clinical Oncology Unit, Faculty of Medicine, University of Malaya, Kuala Lumpur, Malaysia.

E-mail address: nm_ung@um.edu.my (N.M. Ung).
} 
soft tissues sarcoma, prostate and gynecological cancer treatment [11-13].

A relatively new MOSFET-based detector, namely the MOSkin was designed and developed by the Centre for Medical Radiation Physics (CMRP), University of Wollongong, Australia. With its relatively small physical dimensions and active volume, MOSkin had been introduced and tested for IVD in brachytherapy. Previous works on the MOSkin detector has been focused on characterizing and identifying dose measurement for prostate, nasopharyngeal and vaginal HDR brachytherapy with the use of Iridium-192 (Ir-192) as the brachytherapy source [14-21].

Ir-192 is the most commonly-used isotope for HDR brachytherapy. Recent technology has allowed for the application of HDR brachytherapy with the use of Cobalt-60 (Co-60) isotope, with the same dimensions as Ir-192. There are no prominent advantages or disadvantages of Co-60 source with regard to clinical aspects except for the logistical consideration as it requires the source to be exchanged every 5 years, compared to 3-monthly exchanges for Ir-192 [22-24]. The purpose of this study is to characterize the physical properties as well as to evaluate the suitability of MOSkin as an in vivo detector in HDR Co-60 brachytherapy system. This study, to the best of our knowledge, was the first to characterize a MOSkin detector under Co-60 as the HDR brachytherapy source.

\section{Materials and methods}

\subsection{Cobalt-60 HDR brachytherapy system}

An Eckert \& Ziegler BEBIG MultiSource ${ }^{\circledR}$ HDR remote afterloader brachytherapy treatment unit model 1322-0012 (Eckert \& Ziegler, Germany) was used throughout the study. The geometric design, materials and dosimetric properties of BEBIG Cobalt-60 source (model Co0.A86) are described elsewhere [23-26]. The radiation source of Co60 with initial activity of $68.05 \mathrm{GBq}$ calibrated on 11th February 2016 was employed in the study. The source strength was verified locally using a PTW (Physikalisch-Technishe Werkstatten GmbH, Freiburg, Germany) well-type ionization chamber Type 077091 resulting in an agreement of within $\pm 2 \%$.

\subsection{MOSkin dosimetry system}

A patented MOSFET of p-type detector, MOSkin is characterized by its unique packaging which uses a thin, flexible and reproducible polyamide film with $7 \mathrm{mg} / \mathrm{cm}^{2}$ thickness to protect its sensor and acts as the build-up layer of the MOSkin. It is equivalent to the thickness of the skin basal layer in human. It has a physical dimension of approximately $2 \times 1 \times 0.5 \mathrm{~mm}^{3}$ as well as a small sensitive gate oxide volume of $4.8 \times 10^{-6} \mathrm{~mm}^{3}$ and thus is suitable for measurements of doses at steep dose gradient region and for skin dosimetry $[16,18]$. Information on MOSkin has been described in detail elsewhere [14-20,27,28]. The detector is read-out in real time with a frequency of $1 \mathrm{~Hz}$ during treatment delivery by connection to a reader with $+15 \mathrm{~V}$ potential difference across the detector gate oxide during irradiation. The MOSkin operates on the same principle as a conventional MOSFET detector. The voltage that was required to initiate the source-drain current is known as threshold voltage, $\mathrm{V}_{\mathrm{th}}$. The change in threshold voltage, $\Delta \mathrm{V}_{\text {th }}$ across the gate oxide ergo proportional to absorbed dose calculated as $=\mathrm{V}_{\text {th }}$ (post exposure) $-\mathrm{V}_{\text {th }}$ (pre exposure). Fig. 1(a) shows the MOSkin dosimetry system used in this study.

\subsection{Phantoms}

All measurements were performed using a polymethyl methacrylate (PMMA) phantom with a dimension of $300 \times 300 \mathrm{~mm}^{2}$ and overall thickness of $300 \mathrm{~mm}$ except for detectors calibration. A slab of PMMA was specially designed and locally fabricated to house the MOSkin

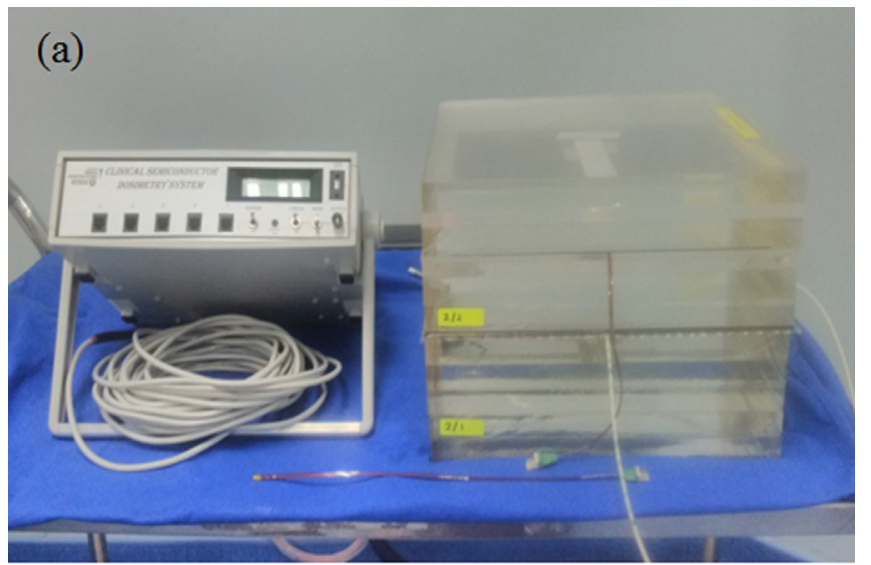

(b)

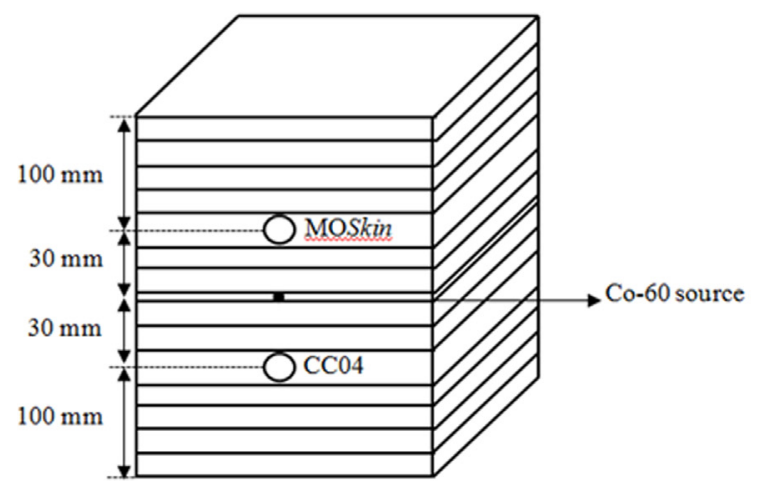

Fig. 1. (a) MOSkin dosimetry system. (b) Set-up of MOSkin calibration procedure under Co-60 source. Both MOSkin and CC01 detectors were aligned parallel at $30 \mathrm{~mm}$ distance to Co-60 source in a solid water phantom.

detector. The solid water phantom (GAMMEX RMI, Middleton, USA) with a slab for insertion of $0.1 \mathrm{cc}$ cylindrical chamber, CC01 (Scanditronix, USA) was used in the detector's calibration.

\subsection{Characterization}

\subsubsection{Calibration}

Calibration under HDR Co-60 brachytherapy source for MOSkin detector was performed by exposing both MOSkin and CC01 simultaneously in the solid water phantom. The source was placed at source to detector distance of $30 \mathrm{~mm}$ as shown in Fig. 1(b). Slabs of $100 \mathrm{~mm}$ thickness were placed above and below the detectors to provide sufficient scattering medium. A plan was generated using the HDRplus treatment planning system (TPS) version 2.6 (Eckert \& Ziegler BEBIG $\mathrm{GmbH}$, Germany) system to deliver $1 \mathrm{~Gy}$ of reference dose at $30 \mathrm{~mm}$ depth. Detectors were irradiated three times to obtain the average readings. The average readings were denoted as $\Delta \mathrm{V}_{\text {mean }}$ for MOSkin detector. Readings from CC01 detector were converted into absolute dose value, $\mathrm{D}_{\mathrm{CC} 01}$ using the TRS 398 photon dose calculation formalism [29]. $\mathrm{D}_{\mathrm{CC} 01}$ in this study was used to verify the AAPM TG43 calculated dose in brachytherapy treatment planning system, $\mathrm{D}_{\text {TPS. Thus, MOSkin' }}$ calibration factor (CF) or sensitivity can be determined either using $\mathrm{D}_{\mathrm{CC} 01}$ or $\mathrm{D}_{\mathrm{TPS}}$ as reference dose data as shown in the equations below:

$C F_{C C 01}=\Delta V_{\text {mean }} / D_{C C 01}$

$C F_{T P S}=\Delta V_{\text {mean }} / D_{T P S}$

The CF obtained from both methods was compared.

\subsubsection{Reproducibility and linearity}

The calibration procedure was repeated for three consecutive days 
and three consecutive weeks to evaluate the reproducibility of the detector. Linearity test was conducted with a similar experimental setup to the calibration procedure. Plans were generated with doses ranging from 1 to $7 \mathrm{~Gy}$ with $1 \mathrm{~Gy}$ intervals, corresponding to the range of intracavitary brachytherapy doses. The resulting MOSkin reading in $\mathrm{mV}$ was plotted against the prescribed dose. Regression analysis was performed to obtain the coefficient of determination $\left(R^{2}\right)$. By averaging the detectors' sensitivity from each dose fraction, MOSkin's sensitivity which has the unit of $\mathrm{mV} \mathrm{cGy}^{-1}$, was calculated and determined from this experiment.

\subsubsection{Angular dependence}

The angular dependence of MOSkin detector was assessed by measuring the responses at various angles in the azimuthal and polar axis due to the possibility of different source beam direction relative to the detector during brachytherapy treatment. Measurements were performed at a distance of $30 \mathrm{~mm}$ from the source with the detector placed in the custom-made PMMA holder inserted into the PMMA slab. For azimuthal axis, measurement of reference angle at $0^{\circ}$ was carried out by ensuring that the active volume of MOSkin directly faced the source. The subsequent measurements were taken by rotating the holder in clockwise position at every $45^{\circ}$ interval.

The polar angular dependence measurement was carried out by moving the Co- 60 source from the first dwell position at the tip of the applicator until reaching the ninth dwell position with $10 \mathrm{~mm}$ separation distance as shown in Fig. 2. The fifth dwell position was fixed perpendicular to the MOSkin active volume and regarded as the reference angle. Readings at each dwell position were corrected for differences for source-detector distance. The corrected MOSkin readings were then compared with doses calculated by TPS to assess the changes in dose measured due to the angular dependence of MOSkin. All readings in both the azimuth and polar angle measurements were normalized to the reading obtained at each respective reference angle.

\subsubsection{Distance dependence}

Distance dependent test was performed to evaluate the variation in MOSkin's sensitivity due to photon energy spectrum changes at varying distance ranging between $15 \mathrm{~mm}$ and $55 \mathrm{~mm}$ with $5 \mathrm{~mm}$ intervals from Co-60 source. The change of distance was accompanied by change in dose rate at each depth. Thus, the variations of sensitivity due to both distance and dose rate can be evaluated simultaneously. This study was performed on PMMA slab phantom as it possessed modest differences in properties compared to water in terms of the density and electron stopping power. The inverse of the detectors' sensitivity for each depth is the distance-dependent correction factor $\left(\mathrm{Dd}_{\mathrm{cf}}\right)$ of the MOSkin detector. Correction factors at each depth were normalized to the maximum depth of $55 \mathrm{~mm}$.

\subsection{In phantom verification}

To verify dose measurements using the MOSkin detector, a simple phantom dose measurement was conducted. A stack of solid water phantoms with a source guide tube and MOSkin detectors embedded

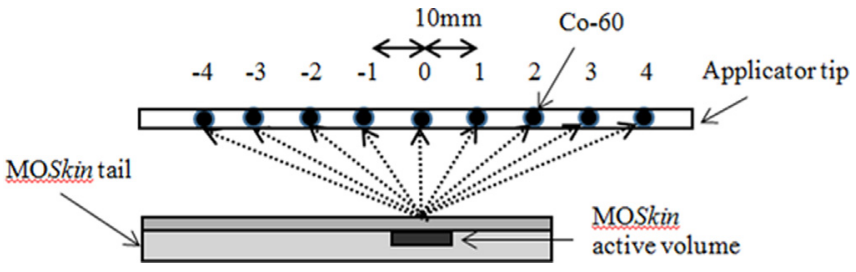

Fig. 2. Source dwelling position from tip of applicator until ninth dwell position along longitudinal axis for measurement of polar angle dependency test. Source at dwell position 0, perpendicular to detector surface defined as reference angle. within the phantom were set up in a similar manner to the distance dependent setup. A detector was placed at every $5 \mathrm{~mm}$ depth interval starting from $15 \mathrm{~mm}$ source-detector distance. The setup and phantom were scanned using a CT simulator (Brilliance CT Big Bore, Philips Healthcare, Andover, MA) using $3 \mathrm{~mm}$ slice thickness. The acquired images were used for planning with a prescribed dose of $1 \mathrm{~Gy}$ at $30 \mathrm{~mm}$ source-detector distance.

Doses were measured at each depth in the solid water phantom from 15 to $55 \mathrm{~mm}$ depth with distance-dependent correction factor incorporated into the dose conversion using the equation below:

Dose $(G y)=\Delta V_{t h} /\left(C F \times D d_{c f}\right)$

The correction factor to account for angular effect was not accounted in the formula as the detector was perpendicular to the source. On TPS, point doses were determined on the image at detector active volume assumed to be located within the phantom. Measured doses were then compared with the TPS calculated dose.

\section{Results}

\subsection{Characterization}

\subsubsection{Calibration}

The calibration factors derived from two methods resulted in small difference with a 0.01 Gy dose deviation as shown in Table 1 . The insignificant difference may be due to the small active volume of CC01, comparable with the small active volume of MOSkin detector. Both detectors are deemed to be suitable for dose measurements in the high dose region of brachytherapy condition. In this study, 3 individual detectors have been used for characterization. For multiple detector usage, it is recommended to calibrate each detector individually regardless of the batch, prior to clinical use, as comparison of calibration factors among three MOSkin detectors resulted in a maximum difference of $8 \%$ above the mean value [19]. The three detectors were calibrated prior to characterization and the maximum sensitivity deviation was $2.5 \%$ as shown in Table 2 .

\subsubsection{Reproducibility and linearity.}

The measurement of MOSkin shows variations of $1.1 \%, 2.6 \%$ and $1.7 \%$ for intra-day, inter-day and weekly reproducibility respectively as shown in Table 3. Fig. 3 shows excellent MOSkin's linearity with $R^{2}=0.9997$ and consistent sensitivity $(\mathrm{mV} / \mathrm{cGy})$ at each dose level with average sensitivity of $2.34 \pm 0.02$ and maximum deviation of $2.5 \%$.

\subsubsection{Angular dependence}

Fig. 4 shows the normalized responses of the MOSkin detector at different azimuth axis angles. The highest response was produced at $0^{\circ}$ angles, when the source was perpendicular to the detector's active volume with 0.015 standard deviation of measurement. A decrease of $0.8 \%$ and $2 \%$ in the MOSkin responses was observed at $45^{\circ}$ and $315^{\circ}$ angles, respectively. There was an approximately $10 \%$ drop in the response when the detector was turned to $\pm 90^{\circ}$. Minimum MOSkin responses were recorded at $\pm 135^{\circ}$ with an approximate drop of $14 \%$.

Table 1

Calibration factors calculated for MOSkin as CC01 and AAPM TG43 TPS dose as reference dose data.

\begin{tabular}{lll}
\hline & \multicolumn{2}{l}{ Reference Dose Data } \\
\cline { 2 - 3 } & CC01 & TPS \\
\hline Dose (Gy) & $0.99^{* a \mathrm{a}}$ & $1.00^{* \mathrm{~b}}$ \\
Calibration factor (mV/cGy) & 2.38 & 2.38 \\
\hline
\end{tabular}

$* \mathrm{a}=$ dose calculated from IAEA TRS-398 formula.

$* \mathrm{~b}=$ AAPM TG43, TPS planning dose. 
Table 2

Measured sensitivity of three MOSkin detectors used in this study.

\begin{tabular}{lll}
\hline $\begin{array}{l}\text { Detector 1 Sensitivity } \\
\left(\mathrm{mV} \mathrm{cGy}^{-1}\right)\end{array}$ & $\begin{array}{l}\text { Detector 2 Sensitivity } \\
(\mathrm{mV} \mathrm{cGy}\end{array}$ & $\begin{array}{l}\text { Detector 3 Sensitivity } \\
(\mathrm{mV} \mathrm{cGy}\end{array}$ \\
\hline 2.38 & 2.35 & 2.41 \\
\hline
\end{tabular}

Table 3

Reproducibility of MOSkin three measurements in a day, three consecutive days (inter-day) and three consecutive weeks (inter-week).

\begin{tabular}{llll}
\hline & \multicolumn{2}{l}{ Readings $(\mathrm{V})$} & \\
\cline { 2 - 4 } & Intra-day & Inter-day & Weekly \\
\hline Measurement 1 & 0.235 & 0.238 & 0.240 \\
Measurement 2 & 0.232 & 0.230 & 0.236 \\
Measurement 3 & 0.230 & 0.242 & 0.232 \\
Mean \pm SD & $0.232 \pm 0.003$ & $0.237 \pm 0.006$ & $0.236 \pm 0.004$ \\
\hline
\end{tabular}

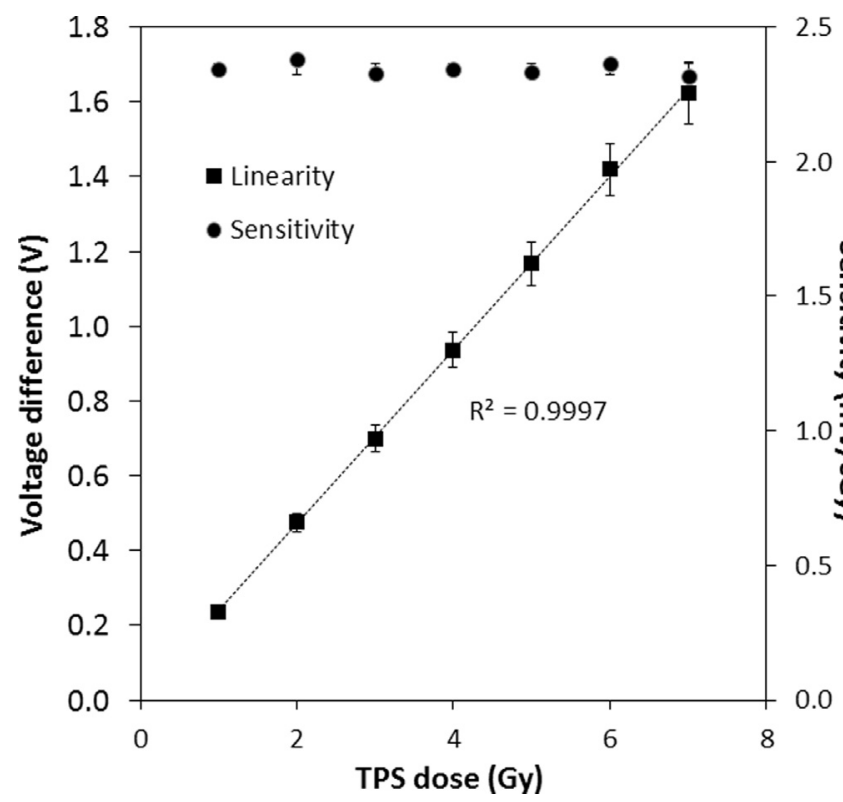

Fig. 3. Response of MOSkin detector as a function of increasing range of intracavitary brachytherapy doses (Gy). The error bars indicate the $2 \%$ measurement uncertainty observed in MOSkin response.

Fig. 5 shows the ratio of MOSkin measured doses over TPS calculated doses at several polar angles normalized to dwell position of zero degree. The results revealed that the measured doses from MOSkin generally agree with the TPS calculated doses to within $\pm 7 \%$, except for a difference of $15 \%$ recorded at polar angle $34^{\circ}$.

\subsubsection{Distance dependence}

In the distance dependent test, the correction factors in Fig. 6 were fitted with a least-squares polynomial function. Consequently, correction factors at other depths in between 15 and $55 \mathrm{~mm}$ depth can be obtained from this graph through interpolation. The normalized correction factors in general decreases with depth, but with small variation of $<2 \%$. From our clinical experience, the distance from OARs to the source from the planning images, within range of $20-50 \mathrm{~mm}$, and thus, the equation on the graph can be used to determine the distance dependent correction factor within this range of depth. Our results were comparable with those reported by Gambarini et al. [30] and Qi et al. [19], in which the correction factors decreased with increasing source to MOSkin detector distance as shown in Fig. 6. It has been reported in a study from our group that the response of normalized MOSkins'

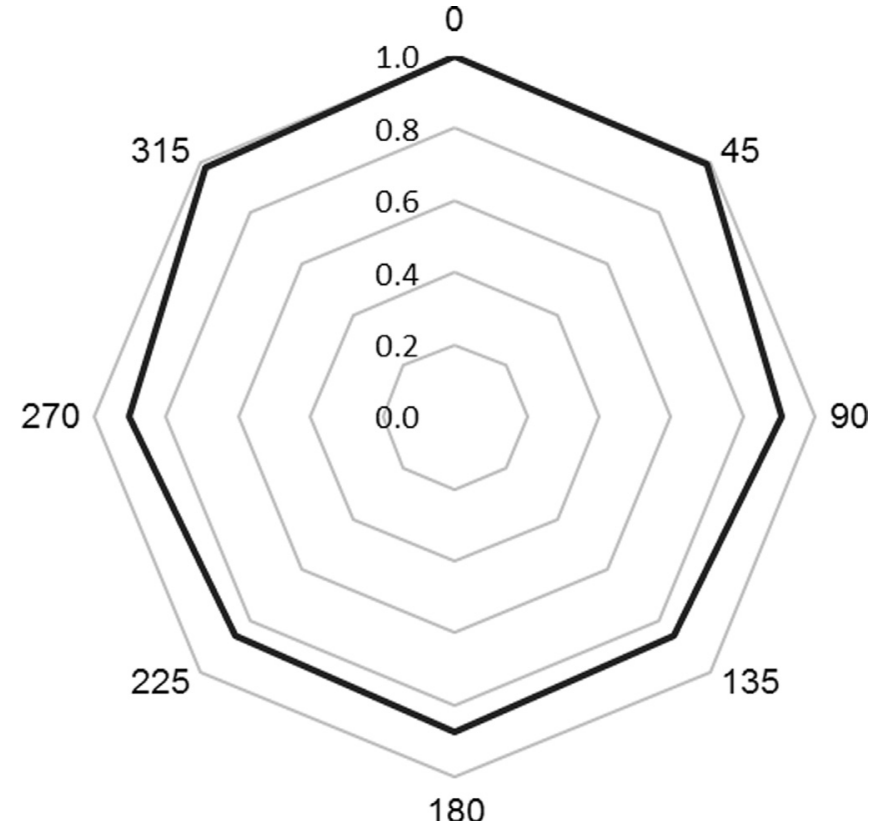

Fig. 4. Detector responses at different rotational angles. All readings were normalized to the reading at $0^{\circ}$.

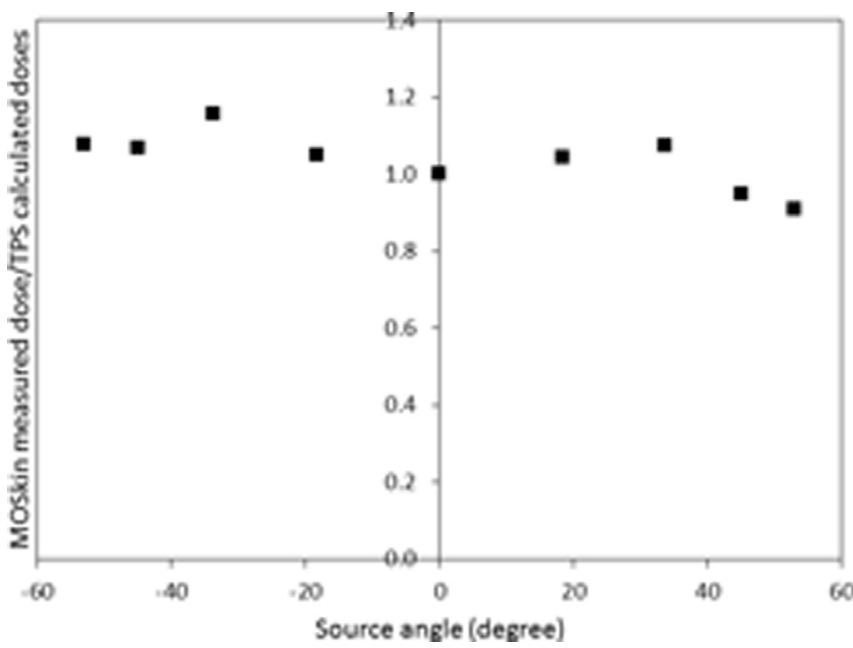

Fig. 5. Ratio of MOSkin measured doses over TPS calculated doses at several polar angles normalized to dwell position of zero degree. Positive dwell positions represent the source movement away from the applicator tip and from detector surface, towards the detector end-tail.

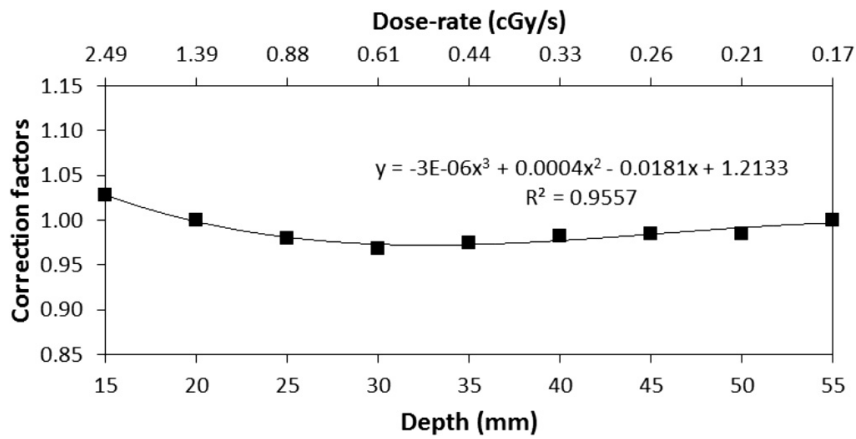

Fig. 6. Relative distance dependent correction factor of MOSkin normalized to maximum depth of $55 \mathrm{~mm}$. 
sensitivity is $<1 \%$ with varied dose-rate [31].

\subsection{In phantom verification}

The decrease of the dose around the source within the source distance range in our study is mainly described by the inverse square law as shown in Fig. 7 with doses at each depth normalized to maximum depth of $55 \mathrm{~mm}$. There were $0.1 \%$ to $\pm 3.0 \%$ differences between MOSkin measurement and doses calculated by TPS. When the discrepancies expressed in terms of absolute dose as shown in Table 5, the dose differences was in the range of $0.01-0.4 \mathrm{~Gy}$ with maximum differences occurred at the smallest distance of $15 \mathrm{~mm}$. The doses measured by MOSkin detectors at depths other than $15 \mathrm{~mm}$ were found to be higher than TPS calculated doses. The MOSkin responses were generally in good agreement with the AAPM TG43 calculated TPS dose and follows the inverse square law.

\subsection{Uncertainty analysis}

The total uncertainty of using MOSkin detector for IVD during brachytherapy is calculated by taking the quadrature sum of individual uncertainty obtained from the characterization tests in this study. By taking into account the uncertainties in each characterization test measurement (1 standard deviation) of; $1.9 \%$ reproducibility, $1 \%$ readout unit, $1.8 \%$ distance-dependence (up to $55 \mathrm{~mm}$ source-detector distance) , 2\% sensitivity (over 1-7 Gy doses range), 1.9\% azimuthangle dependence $\left( \pm 90^{\circ}\right)$ and $3.2 \%$ polar-angle dependence $\left( \pm 45^{\circ}\right)$, the overall intrinsic uncertainties of MOSkin $(k=1)$ is $5.1 \%$. By adding the uncertainties of TPS dose calculation for high-energy sources of $2.6 \%$ and source calibration uncertainty of $1.5 \%$ produced by the AAPM TG-138 and GEC-ESTRO guidelines [32], the overall MOSkin uncertainty $(k=1)$ budget in this study for IVD in brachytherapy was $\pm 5.9 \%$

\section{Discussion}

\subsection{Calibration, reproducibility and linearity}

The choice of using either CC01 or TPS as dose reference data for MOSkin dose calibration factors is rarely discussed in the literature. However, we prefer to apply the CF derived from TPS as reference dose data for conversion of detector response to absorbed dose water in medium, similar to other studies $[14,15,18]$. In addition, Monte Carlo dosimetric studies have been performed and validated on the brachytherapy system used in this study, BEBIG Co-60 HDR by Ballester et al. and Campos and Almeida where the dosimetric parameters were consistent with consensus data set and other published data [26,33].

MOSkin's reproducibility in our study was higher in comparison with published results under $6 \mathrm{MV}$ Linac with deviations of $<1 \%$ for dose ranging from $0 \mathrm{~Gy}$ to $3 \mathrm{~Gy}$ [28]. Qi et al. tested the MOSkin's reproducibility for two read-out modes; manual and real-time for increasing dose ranges of 0.2 to $0.5 \mathrm{~Gy}$ and reported improved reproducibility with increasing dose, with standard deviation of $<2 \%$ at $0.5 \mathrm{~Gy}$. [20]. This indicates excellent stability of MOSkin readout and measurements.

MOSkin's sensitivity from the linearity test was found to be consistent with and within the range of other published results under different radiation sources and beam energies, as shown in Table 4 $[20,27,28]$. The slight differences in sensitivity values were probably due to the intrinsic characteristics of the structural and packaging material that differed between each detector's production [18].

\subsection{Angular dependence}

Larger discrepancies in the detector response at various azimuthal angles in comparison with other published studies may be due to
Table 4

Average sensitivity of MOSkin in comparison with other published studies under different calibration sources.

\begin{tabular}{lll}
\hline Studies & Sensitivity (mV/cGy) & Calibration source \\
\hline Qi et al., 2012 & $2.17 \pm 0.01$ & Iridium-192 \\
Kwan et al., 2014 & 2.49 & 6MV Linac \\
Gambarini et al., 2014 & 2.63 & Iridium-192 \\
Tenconi et al., 2014 & $2.43 \& 2.49$ & Iridium-192 \\
Jong et al., 2014 & $2.53 \pm 0.03$ & 6MV Linac \\
Jong et al., 2017 & $2.49 \pm 0.06$ & 6MV SRS Linac \\
This study & $2.34 \pm 0.02$ & Cobalt-60
\end{tabular}

Table 5

Percentage differences of MOSkin measured and TPS calculated dose in phantom measurements normalized to maximum depth of $55 \mathrm{~mm}$.

\begin{tabular}{llll}
\hline Depth (mm) & TPS dose (Gy) & MOSkin dose (Gy) & Differences (\%) \\
\hline 15 & 14.64 & 14.24 & -2.74 \\
20 & 8.18 & 8.18 & 0.07 \\
25 & 5.18 & 5.28 & 2.03 \\
30 & 3.57 & 3.69 & 3.26 \\
35 & 2.61 & 2.67 & 2.56 \\
40 & 1.96 & 2.00 & 1.82 \\
45 & 1.54 & 1.56 & 1.6 \\
50 & 1.25 & 1.27 & 1.56 \\
\hline
\end{tabular}

differences in the Co-60 spectrum. Qi et al. determined the MOSkin response for azimuth angular dependence under Ir-192 source to vary less than $2 \%$ over within $360^{\circ}[17,19]$. The present azimuth angle dependence results, however, were in close agreement with the study by Jong et al. [28] when comparing the detector response used in facedown orientation under $6 \mathrm{MV}$ photon beam with $180^{\circ}$ angular response in our study. A deviation of $>10 \%$ in the range of beam incident angle from $0^{\circ}$ to $75^{\circ}$ was found in this study with maximum deviation of $18 \%$ at $75^{\circ}$ angle [28]. At $180^{\circ}$ angle, the photon spectrum changed as the water-equivalent depth (WED) of MOSkin detector increased to approximately $0.9 \mathrm{~mm}$ (instead of $0.07 \mathrm{~mm}$ ) due to the silicon substrate thickness under the oxide gate. Due to the steep dose gradient in brachytherapy dose distribution, a sub-millimeter change in detector WED will result in significant changes in the detector response.

In polar angular dependency test, the corrected MOSkin readings for $\pm 53^{\circ}$ angle from the surface of detector shown to follow the trend of TPS calculated dose. It was observed that the measured dose increases for dwell position 2 to position-4, towards the MOSkin tail. The variation within the range of measured angle in our study found to be higher in comparison with study by Qi et al., with a sensitivity variation of within $2 \%$ for $\pm 60^{\circ}[19]$. This can be reduced if dose measurement performed at $\pm 20^{\circ}$ angle in which the variation is $<3 \%$.

\subsection{Distance dependence}

Determination of distance-dependent correction factor had shown comparable results with other studies of lower correction factor with increased distance $[15,20]$. In these published studies, either single or dual MOSkins were shown to have significant increase in correction factors at decreasing source-detector distance ranging from 7 to $50 \mathrm{~mm}$. However, at depth of $\geq 45 \mathrm{~mm}$, a slight increase of correction factors until maximum depth of $55 \mathrm{~mm}$ was observed in our study. This may be due to inherent uncertainties of read-out unit as the response of MOSkin detector at depth of $\geq 45 \mathrm{~mm}$ was $<100 \mathrm{mV}$. This uncertainty could have been reduced to $\pm 1 \%$ for $>100 \mathrm{mV}$ reading [34].

\subsection{In phantom verification}

The relatively lower MOSkin response at a smaller distance of $15 \mathrm{~mm}$ and slightly higher measured doses at greater distances, as 


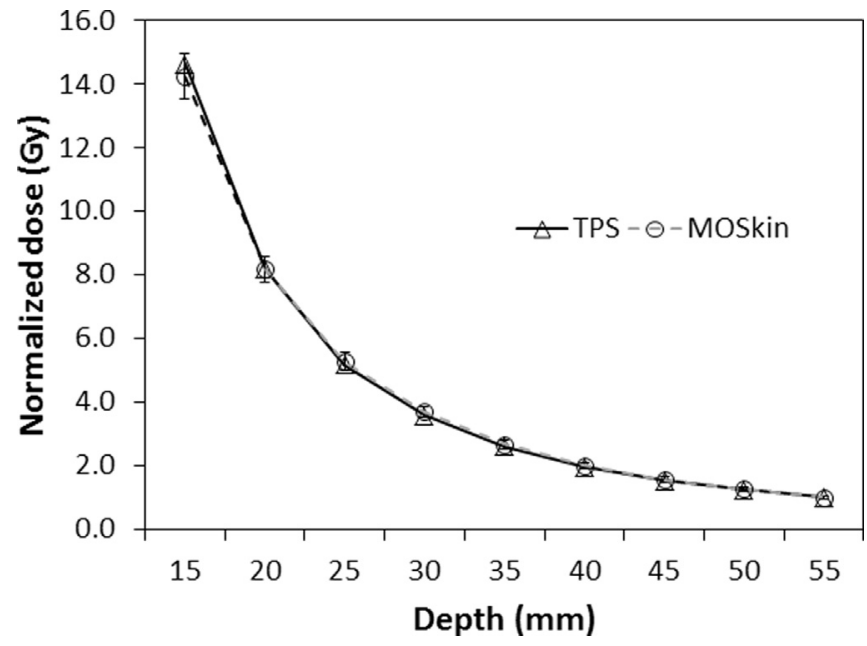

Fig. 7. Comparison of measured dose from MOSkin detector with calculated dose of TPS. Readings were normalized to maximum depth of $55 \mathrm{~mm}$. Measurements uncertainty is indicated by $5 \%$ error bars. The large differences at smaller source-to-detector distance showed the importance of accurate positioning and thus should be avoided for clinical measurement.

shown in Fig. 7, is possibly due to some uncertainties. Two types of uncertainties are expected to arise: a) detector positioning, b) detector response. The uncertainties due to detector positioning dominate for measurements close to the source while the detector response affected reading at long distances with minimum of combined uncertainties occur at $25 \mathrm{~mm}$ source distance [35].

At smaller distance, as the detector is located within the strong dose gradient, the dose reduction after the first $10 \mathrm{~mm}$ is around $50 \%$ near the source [36]. This is consistent with our measurements, with nearly $60 \%$ dose fall off from measurements at distances of $15-25 \mathrm{~mm}$. Due to this, a $1 \mathrm{~mm}$ detector positioning error can result in large dose differences at small distances. The uncertainties in dose measurement at larger source to detector distance arise mainly due to the uncertainties in detectors response which can be attributed by lower dose delivered at larger distances. In this study, dose of $1 \mathrm{~Gy}$ was delivered at calibration depth of $30 \mathrm{~mm}$. Following the inverse square law, the dose at subsequent distance beyond the calibration depth will be further decreased with inversely proportional to the distance $\left(1 /\right.$ distance $\left.^{2}\right)$. As the MOSkin readout unit uncertainty reduced to $\pm 1 \%$ for $\Delta \mathrm{V}_{\text {th }}$ greater than $100 \mathrm{mV}$, hence the dose measurement uncertainty will become more significant for lower dose [34].

The stopping powers data retrieved from National Institute of Standards and Technologies (NIST), for PMMA and water under $1.25 \mathrm{MeV}$ photon energy were 1.781 and $1.845 \mathrm{MeV} \mathrm{cm}^{2} / \mathrm{g}$ respectively. With modest difference of $3.4 \%$, one can assumed that the measured doses in PMMA should be comparable with dose measurements in water. In addition, the absorbed-dose water equivalence was found to be less dependent on phantom material for high-energy brachytherapy sources such as Ir-192 and Co-60 and thus PMMA can be used with correction factors applied [37]. The dose deviation also contributed by inaccurate determination of detector location at TPS. The deviation of detector position during treatment from the assumed location determined on TPS in a study by Romanyukha et al., hence leads to $\pm 3.62 \%$ dose indicated by TPS [38].

\subsection{Application of MOSkin for in-vivo dosimetry}

The difference in temperature at the time of MOSkin calibration and patients' body temperature during measurement has been proven to be negligible as no significant changes have been noted for the same type of MOSFET-based detector sensitivity within a temperature range of $15^{\circ}-40^{\circ}$ given that the detector reached thermal equilibrium [39].
Hence no temperature correction factor has been determined in our study of MOSkin characterization as we allowed detectors to reach thermal equilibrium for approximately $60 \mathrm{~s}$ before any measurement.

To eliminate uncertainties in detector positioning and electronic equilibrium of dose distribution, measurements of dose with the use of Co-60 brachytherapy were performed at a source-detector distance $>$ $10 \mathrm{~mm}$. Ballester et al. found that at $1 \mathrm{~mm}$ from Ir-192 source, electrons contributed to $9.4 \%$ of the total dose while collisional kerma equals to total dose occurring at $2 \mathrm{~mm}$ source distance [40]. In comparison to Ir192, at the same distance of $1 \mathrm{~mm}$, only $1.9 \%$ electron contribution was found for Co-60 with collisional kerma equivalent to total dose at larger source distance of $7 \mathrm{~mm}$ [40]. Thus, electronic disequilibrium occurs at larger distances for Co-60, compared with Ir-192 source. In view of this, it is recommended that any dose measurement with the use of Co-60 source should be performed at source detector distance $>10 \mathrm{~mm}$.

At larger distance, the measured detector response can be converted into absorbed dose by applying the correction factors determined from tests above. The measured response is proportional to the summation of the contribution from all dwell positions, with distance and angle measured with respect to detector position defined on simulation images Assuming no detector movement throughout the imaging and treatment procedures, the distance and angle can be determined from three-dimensional images (CT or MRI).

\section{Conclusion}

In this work, MOSkin was tested for its suitability as a detector for in-vivo dosimetry in HDR Co-60 brachytherapy due to its special characteristic of being small in size. Characterization of MOSkin revealed good linearity with flat sensitivity within the clinical brachytherapy dose range and good reproducibility over time. Sensitivity variation as function of distance-dependent correction factors need to be taken into account when converting the response into absorbed dose. Thus, MOSkin can be considered to be a good detector of choice for in-vivo dosimetry in HDR Co-60 intracavitary brachytherapy.

\section{Acknowledgment}

This work was supported by Postgraduate Research Grant (PPP) Project No. PG211-2015B, Faculty of Medicine, University of Malaya. The authors would like to thank staffs of the Department of Clinical Oncology, University of Malaya Medical Centre for the assistance and support of this study.

\section{References}

[1] Georg P, Kirisits C, Goldner G, Dorr W, Hammer J, Potzi R, et al. Correlation of dosevolume parameters, endoscopic and clinical rectal side effects in cervix cancer patients treated with definitive radiotherapy including MRI-based brachytherapy. Radiother Oncol 2009;91:173-80. https://doi.org/10.1016/j.radonc.2009.01.006.

[2] Koom WS, Sohn DK, Kim J-Y, Kim JW, Shin KH, Yoon SM, et al. Computed tomography-based high-dose-rate intracavitary brachytherapy for uterine cervical cancer: preliminary demonstration of correlation between dose-volume parameters and rectal mucosal changes observed by flexible sigmoidoscopy. Int J Radiat Oncol Biol Phys 2007;68:1446-54. https://doi.org/10.1016/j.ijrobp.2007.02.009.

[3] Jursinic PA. Characterization of optically stimulated luminescent dosimeters, OSLDs, for clinical dosimetric measurements. Med Phys 2007;34:4594-604. https://doi.org/10.1118/1.2804555.

[4] Andersen CE, Nielsen SK, Greilich S, Helt-Hansen J, Lindegaard JC, Tanderup K. Characterization of a fiber-coupled Al2O3: C luminescence dosimetry system for online in vivo dose verification during 192Ir brachytherapy. Med Phys 2009;36:708-18. https://doi.org/10.1118/1.3063006.

[5] Sharma S. Vendor Development Process in Automobile Industry in India: A Comparative Study 2013;1:118-24.

[6] Cartwright LE, Suchowerska N, Yin Y, Lambert J, Haque M, McKenzie DR. Dose mapping of the rectal wall during brachytherapy with an array of scintillation dosimeters. Med Phys 2010;37:2247. https://doi.org/10.1118/1.3397446.

[7] Ghahramani F, Allahverdi M, Jaberi R. Dependency of semiconductor dosimeter responses, used in MDR/LDR brachytherapy, on factors which are important in clinical conditions. Reports Pract Oncol Radiother 2008;13:29-33. https://doi.org/ 10.1016/S1507-1367(10)60079-X. 
[8] Alecu R, Alecu M. In-vivo rectal dose measurements with diodes to avoid misadministrations during intracavitary high dose rate brachytherapy for carcinoma of the cervix. Med Phys 1999;26:768-70. https://doi.org/10.1118/1.598598.

[9] Price RA, Benson C, Joyce MJ, Rodgers K. Development of a RadFET linear array for intracavitary in vivo dosimetry during external beam radiotherapy and brachytherapy. IEEE Trans Nucl Sci 2004;51:1420-6. https://doi.org/10.1109/TNS. 2004.832570.

[10] Zaman ZK, Ung NM, Malik RA, Ho GF, Phua VCE, Jamalludin Z, et al. Comparison of planned and measured rectal dose in-vivo during high dose rate Cobalt-60 brachytherapy of cervical cancer. Phys Medica 2014;30:980-4. https://doi.org/10. 1016/j.ejmp.2014.07.002.

[11] Van Gellekom MPR, Canters RAM, Dankers FJWM, Loopstra A, van der SteenBanasik EM, Haverkort MAD. In vivo dosimetry in gynecological applications-a feasibility study. Brachytherapy 2016. https://doi.org/10.1016/j.brachy.2017.04. 240.

[12] Melchert C, Soror T, Kovács PG. Quality assurance during interstitial brachytherapy: in vivo dosimetry using MOSFET dosimeters 2018;10:6-11.

[13] Ramapandian S, Nagarajan V, Mukherji A. Inter-Fraction and Intra-Fraction Variation in the Absorbed-Dose Delivery during Interstitial High Dose Rate Brachytherapy — A Study Using 2017:445-56. doi:10.4236/ijmpcero.2017.64040.

[14] Carrara M, Tenconi C, Rossi G, Borroni M, Cerrotta A, Grisotto S, et al. In vivo rectal wall measurements during HDR prostate brachytherapy with MOSkin dosimeters integrated on a trans-rectal US probe: Comparison with planned and reconstructed doses. Radiother Oncol 2016;118:148-53. https://doi.org/10.1016/j.radonc.2015. 12.022 .

[15] Gambarini G, Carrara M, Tenconi C, Mantaut N, Borroni M, Cutajar D, et al. Online in vivo dosimetry in high dose rate prostate brchytherapy with MOSkin detectors: in phantom feasibility study. Appl Radiat Isot 2014;83(Pt C):222-6. https://doi.org/ 10.1016/j.apradiso.2013.06.001.

[16] Hardcastle N, Cutajar DL, Metcalfe PE, Lerch MLF, Perevertaylo VL, Tomé WA, et al. In vivo real-time rectal wall dosimetry for prostate radiotherapy. Phys Med Biol 2010;55:3859-71. https://doi.org/10.1088/0031-9155/55/13/019.

[17] Ian K, Andrew H, Michael L, Bongsoo L, Chin YS, Bucci J, et al. Measurement of rectal dose during HDR brachytherapy using the new MO skin dosimeter. J Nucl Sci Technol 2008;45:481-4. https://doi.org/10.1080/00223131.2008.10875895.

[18] Tenconi C, Carrara M, Borroni M, Cerrotta A, Cutajar D, Petasecca M, et al. TRUSprobe integrated MOSkin detectors for rectal wall in vivo dosimetry in HDR brachytherapy: In phantom feasibility study. Radiat Meas 2014;71:379-83. https:// doi.org/10.1016/j.radmeas.2014.05.010.

[19] Qi Z, Deng X, Huang S, Lu J, Lerch M, Cutajar D, et al. Verification of the plan dosimetry for high dose rate brachytherapy using metal-oxide-semiconductor field effect transistor detectors. Int J Med Phys Res Pract 2007;34:2007-13. https://doi. org $/ 10.1118 / 1.2736288$.

[20] Qi Z-Y, Deng X-W, Cao X, Huang S-M, Lerch M, Rosenfeld A. A real-time in vivo dosimetric verification method for high-dose rate intracavitary brachytherapy of nasopharyngeal carcinoma. Med Phys 2012;39:6757-63. https://doi.org/10.1118/ 1.4758067.

[21] Carrara M, Romanyukha A, Tenconi C, Mazzeo D, Cerrotta A, Borroni M, et al. Clinical application of MOSkin dosimeters to rectal wall in vivo dosimetry in gynecological HDR brachytherapy. Phys Medica 2017;41:5-12. https://doi.org/10. 1016/j.ejmp.2017.05.003.

[22] Niatsetski Y, Andrássy M, Pérez-Calatayud J. Co-60 versus Ir-192 in HDR brachytherapy: scientific and technological comparison. Rev Física Médica 2012;13:125-30.

[23] Richter J, Baier K, Flentje M. Comparison of 60 cobalt and 192 iridium sources in high dose rate afterloading brachytherapy. Strahlentherapie Und Onkol 2008;184:187-92.
[24] Strohmaier S, Zwierzchowski G. Comparison of (60)Co and (192)Ir sources in HDR brachytherapy. J Contemp Brachytherapy 2011;3:199-208. https://doi.org/10. 5114/jcb.2011.26471.

[25] Palmer A, Mzenda B. Performance assessment of the BEBIG MultiSource ${ }^{\circledast}$ high dose rate brachytherapy treatment unit. Phys Med Biol 2009;54:7417-34. https://doi. org/10.1088/0031-9155/54/24/011.

[26] Ballester F, Granero D, Pérez-Calatayud J, Casal E, Agramunt S, Cases R. Monte Carlo dosimetric study of the BEBIG Co-60 HDR source. Phys Med Biol 2005;50:N309-16. https://doi.org/10.1118/1.1997712.

[27] Jong WL, Ung NM, Vannyat A, Jamalludin Z, Rosenfeld A, Wong JHD. "Edge-on" MOSkin detector for stereotactic beam measurement and verification. Phys Med 2017;33:127-35. https://doi.org/10.1016/j.ejmp.2016.12.020.

[28] Jong WL, Wong JHD, Ung NM, Ng KH, Ho GF, Cutajar DL, et al. Characterization of MOSkin detector for in vivo skin dose measurement during megavoltage radiotherapy. J Appl Clin Med Phys 2014;15:120-32. https://doi.org/10.1120/jacmp. v15i5.4869.

[29] IAEA TRS. 398. Absorbed dose determination in external beam radiotherapy: An International Code of Practice for Dosimetry based on standards of absorbed dose to water. Vienna Int At Energy Agency 2000.

[30] Gambarini G, Carrara M, Tenconi C, Mantaut N, Borroni M, Cutajar D, et al. Online in vivo dosimetry in high dose rate prostate brchytherapy with MOSkin detectors: In phantom feasibility study. Appl Radiat Isot 2014;83:222-6. https://doi.org/10. 1016/j.apradiso.2013.06.001.

[31] Safari MJ, Wong JHD, Ng KH, Jong WL, Cutajar DL, Rosenfeld AB. Characterization of a MOSkin detector for in vivo skin dose measurements during interventional radiology procedures Characterization of a MO Skin detector for in vivo skin dose measurements during interventional radiology procedures. Med Phys 2015:2550. https://doi.org/10.1118/1.4918576.

[32] DeWerd LA, Ibbott GS, Meigooni AS, Mitch MG, Rivard MJ, Stump KE, et al. A dosimetric uncertainty analysis for photon-emitting brachytherapy sources: Report of AAPM Task Group No. 138 and GEC-ESTRO. Med Phys 2011;38:782-801. https://doi.org/10.1118/1.3533720.

[33] Campos LT, De Almeida CEV. Monte Carlo dosimetry of the 60Co BEBIG high dose rate for brachytherapy. PLoS One 2015;10:1-9. https://doi.org/10.1371/journal. pone.0139032.

[34] Kwan IS, Wilkinson D, Cutajar D, Lerch M, Rosenfeld A, Howie A, et al. The effect of rectal heterogeneity on wall dose in high dose rate brachytherapy. Int $\mathrm{J}$ Med Phys Res Pract 2009;36:224-32. https://doi.org/10.1118/1.3031111.

[35] Andersen CE, Nielsen SK, Lindegaard JC, Tanderup K. Time-resolved in vivo luminescence dosimetry for online error detection in pulsed dose-rate brachytherapy. Med Phys 2009;36:5033-43. https://doi.org/10.1118/1.3238102.

[36] Rivard Mark J, Beaulieu Luc, Thomadsen Bruce R. Clinical brachytherapy physics. Medical Physics Publishing, Inc.; 2017.

[37] Perez-Calatayud J, Ballester F, Das RK, Dewerd LA, Ibbott GS, Meigooni AS, et al. Dose calculation for photon-emitting brachytherapy sources with average energy higher than $50 \mathrm{keV}$ : Report of the AAPM and ESTRO. Med Phys 2012;39:2904-29. https://doi.org/10.1118/1.3703892.

[38] Romanyukha AA, Carrara M, Tenconi C, Mazzeo D, Rossi G, Borroni M, et al. Applications of MOSkin dosimeters for quality assurance in gynecological HDR brachytherapy: an in-phantom feasibility study. Radiat Meas 2016. https://doi.org/ 10.1016/j.radmeas.2016.12.010.

[39] Cheung T, Butson MJ, Yu PKN. Effects of temperature variation on MOSFET dosimetry. Phys Med Biol 2004;49:N191-6. https://doi.org/10.1088/0031-9155/49/ 13/N02.

[40] Ballester F, Granero D, Pérez-Calatayud J, Melhus CS, Rivard MJ. Evaluation of high-energy brachytherapy source electronic disequilibrium and dose from emitted electrons. Med Phys 2009;36:4250-6. https://doi.org/10.1118/1.3194754. 